\title{
Considering the $\kappa-\mu$ fading channels adopted in multiple antennas downlink non-orthogonal multiple access
}

\author{
Chi-Bao Le ${ }^{1}$, Hong-Nhu Nguyen ${ }^{2,3}$, Ngoc-Long Nguyen ${ }^{2}$, Miroslav Voznak $^{2}$, Nhan Duc Nguyen ${ }^{4}$ \\ ${ }^{1}$ Faculty of Electronics Technology, Industrial University of Ho Chi Minh City (IUH), Ho Chi Minh City, Vietnam \\ ${ }^{2}$ Faculty of Electrical and Computer Science, VSB-Technical University of Ostrava, Ostrava, Czech Republic \\ ${ }^{3}$ Faculty of Electronics and Telecommunications, Saigon University, Ho Chi Minh City, Vietnam \\ ${ }^{4}$ Innovation Center, Van Lang University, Ho Chi Minh City, Vietnam
}

\begin{tabular}{|c|c|}
\hline Article Info & ABSTRACT \\
\hline Article history: & \multirow{12}{*}{$\begin{array}{l}\text { Massive connectivity and effective spectrum usage have become more important as the } \\
\text { use of wireless communication devices and networks has grown dramatically. The ap- } \\
\text { proach of non-orthogonal multiple access (NOMA) is advocated as a viable solution } \\
\text { for meeting consumers' current needs. The signals are overlaid with various power } \\
\text { levels for each user in a NOMA-assisted system, and then broadcast to the receiver. } \\
\text { SIC (successive interference cancellation) is used by the receiver to discriminate and } \\
\text { get the needed signal. Until far, most studies have concentrated on SIC with ideal } \\
\text { features, with only a handful focusing on SIC with imperfect qualities (ipSIC). While } \\
\text { the perfect SIC (pSIC) represents the ideal condition of no data loss and no external } \\
\text { sounds, the ipSIC represents data transfer in a real-time context. In this research, we } \\
\text { will assess the system performance metrics of the investigated NOMA system in the } \\
\text { presence of ipSIC and compare them to the performance of the same user's pSIC. We } \\
\text { define channels as } \kappa-\mu \text { fading distributions, which is more essential. For two desti- } \\
\text { nations, we construct accurate outage probability formulas. Meanwhile, Monte-Carlo } \\
\text { simulations are run to ensure that the mathematical expressions derived are genuine. }\end{array}$} \\
\hline Received Jul 22, 2021 & \\
\hline Revised Nov 21, 2021 & \\
\hline Accepted Dec 22, 2021 & \\
\hline Keywords: & \\
\hline Decode-and-forward & \\
\hline Imperfect successive & \\
\hline interference cancellation & \\
\hline Non-orthogonal multiple & \\
\hline access & \\
\hline Outage probability & \\
\hline & \\
\hline
\end{tabular}

This is an open access article under the CC BY-SA license.

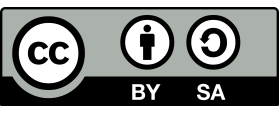

\section{Corresponding Author:}

Nhan Duc Nguyen

Innovation Center, Van Lang University, Ho Chi Minh City, Vietnam

Email: nhan.nd@vlu.edu.vn

\section{INTRODUCTION}

The increasing appetite for the internet of things (IoT) and mobile internet is behind the design push for innovative future wireless communication networks that satisfy high spectral and connectivity efficiency [1]-[3]. Non-orthogonal multiple access (NOMA) is one of the technologies proposed to address spectral efficiency and mass connectivity challenges in future network systems [1]-[3]. Unlike existing orthogonal multiple access (OMA), NOMA accommodates more users by utilizing non-orthogonal resource allocation [1]-[8]. NOMA is divided in two sub-categories: power and code-based NOMA [1], [3], [5]. In this study, we have mainly concerned with power-domain NOMA which typically serves multiple users by grouping and superposing users' data signals at the transmitters. Users experiencing dissimilar channel conditions are usually grouped. Hence, far users experiencing poor channel conditions are allocated greater transmit power and in each group, while the near receiver utilizes successive interference cancellation (SIC) to decode the data transmitted signal to the far user. SIC helps to eliminate interference, thus, users with better channel gain do not require high transmit power to achieve good throughput [9].

Several studies have investigated the performance benefits of power-domain in various NOMA-assisted 
communication networks. For example, [10] studied the outage of NOMA assisted downlink multi-cell millime ter-wave (mmWave) network under Nakagami- $m$ fading. The authors numerically proved that NOMA outperforms OMA in multi-cell mmWave systems. Zhang et al. in [11], the authors demonstrated the individual outage rate of NOMA's fading and power split configurations in cognitive hybrid satellite-ground networks reliant on amplify and forward (AF) procedures. In particular, the ground network channels followed Nakagami- $m$ distribution whilst the satellite links followed generalized shadowed-Rician fading. Differently, the authors in [12], compared the outage and throughput performance of two popular NOMA decoding techniques, SIC and joint-decoding (JD), in uplink machine-to-machine (M2M) communications. Numerical results showed that SIC has similar performance to JD when transmitting powers and traffic load over frequency-flat block-fading multiple access channels are low but underperforms under higher transmit powers or frequency-selectivity conditions. In similar work, in [13], the authors confirmed that NOMA outperforms OMA in enhancing the spectral efficiency of K-tier heterogeneous networks (HetNets).

Another key technology has already deployed in long term evolution (LTE-advanced) and $5 \mathrm{G}$ is massive multiple-input multiple-output (MIMO) [14], [15]. In this work, they examined the utilization of powerdomain NOMA at base stations with multiple antennas. Various works on multiple antennas aided NOMA have focused on perfect SIC such as [16]-[26] whereas only a few have focused on imperfect SIC (ipSIC) as in [27]-[29]. While the perfect SIC (pSIC) represents the ideal condition of no data loss and no external sounds, the ipSIC represents data transfer in a real-time context.

Motivated by recent studies, just a few works have examined how single-input single-output (SISO) downlink NOMA systems perform in general fading channels. For instance, in [30], the authors investigated the impact of residual transceiver hardware impairments on cooperative SISO NOMA under generic $\alpha-\mu$ fading channels. The authors also proposed exact asymptotic formulas for outage probability (OP), ergodic capacity, and energy efficiency to characterize the system performance. Le et al. in [31], the authors studied the uplink performance of SISO NOMA systems subject to imperfect SIC and delay constraints over a wide range of fading composite fading channels like lognormal-Nakagami- $m, K_{G}, \eta-\mu$, Nakagami- $q$ (Hoyt), $\kappa-\mu$, Nakagami- $n$ (Rician), Nakagami- $m$, and Rayleigh fading channels. The authors derived exact expressions of OP, throughput, and capacity based on Meijer-G functions. Moreover, [32], the authors considered OP for downlink and uplink multi-user SISO power-domain NOMA network where users can be subject to one of the fading distributions mentioned in [31]. ElHalawany et al. in [33], the authors also developed exact expressions for ergodic capacity, OP and bit error rate (BER) for a two-user NOMA-aided SISO downlink framework under $\kappa-\mu$ shadowing.

Differently, this paper provides a scheme to highlight the different OP performance of two users separated by an obstruction in a multiple-input single-output (MISO) NOMA network utilizing perfect and imperfect SIC under $\kappa-\mu$ fading channels. Our contributions are listed is being as: We investigate powerdomain NOMA by examining the downlink OP performance under $\kappa-\mu$ fading channels using perfect and imperfect SIC. We formulate exact OP formulas subject to the power allocation coefficients constraints. We evaluate outage under $\kappa-\mu$ fading channels to show how perfect and imperfect SIC guarantees operation in the downlink when there is an obstruction between the two users. Simulation results demonstrate that imperfect $\mathrm{SIC}$, in terms of the OP, outperforms perfect SIC.

The remainder of this document is formatted is being as. The downlink power-domain NOMA with generalized channels is described in section 2. In part 3, we look at the outage performance of a power-domain NOMA scenario. In part 4, we present comprehensive numerical simulations, and section 5 ends the article.

\section{DESCRIPTION OF DOWNLINK NOMA WITH GENERALIZED CHANNELS}

Figure 1 shows a base station (BS) equipped with $N$ antennas to serve two NOMA users, $U_{1}$ and $U_{2}$. Particularly, we assume that the BS is equipped with $n$ antennas in which $n=[1,2, \ldots, N]$. The channel gain of links $B S-U_{1}$ and $B S-U_{2}$ are defined as $g_{n, 1}$ and $g_{n, 1}$, respectively. In this study, the $B S$ transmits the superposed information $s=\sqrt{P b_{1}} s_{1}+\sqrt{P b_{2}} s_{2}$ to the users $\left(U_{1}\right.$ and $U_{2}$ ), where $s_{1}$ and $s_{2}$ are $U_{1}$ and $U_{2}$ the messages, respectively. $b_{1}$ and $b_{2}$ are the power allocation coefficients subject to $b_{2}>b_{1}$ and $b_{2}+b_{1}=1$. $P$ is the $B S$ transmit power. Moreover, $U_{1}$ and $U_{2}$ receive the superposed signal and loop interference signal simultaneously. The observations at $U_{1}$ and $U_{2}$ are [34]:

$$
y_{n, 1}=g_{n, 1}\left(\sqrt{P b_{1}} s_{1}+\sqrt{P b_{2}} s_{2}\right)+\omega_{n, 1},
$$




$$
y_{n, 2}=g_{n, 2}\left(\sqrt{P b_{1}} s_{1}+\sqrt{P b_{2}} s_{2}\right)+\omega_{n, 2}
$$

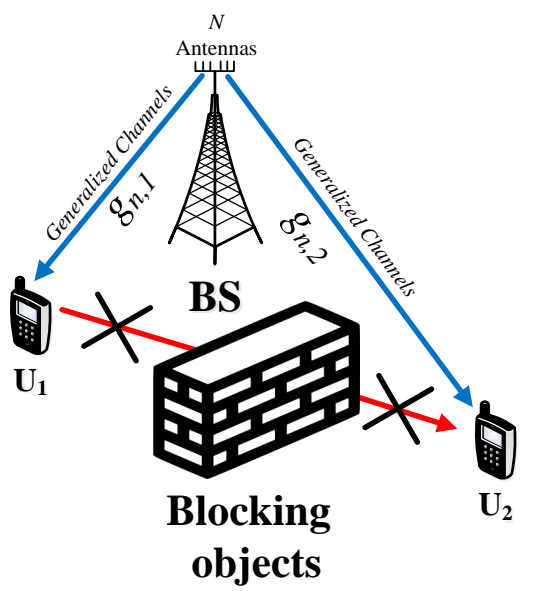

Figure 1. Downlink NOMA with generalized channels

where $\omega_{n, i} \sim \mathcal{C N}\left(0, N_{0}\right)$, for $i=1,2$ is the additive complex white gaussian noise (AWGN).

As given (2), $U_{2}$ is able to detect $s_{2}$ by treating $s_{1}$ in (2) as an interference. The instantaneous signalto-interference-plus-noise ratio (SINR) at $U_{2}$ from (2) is given as:

$$
\tilde{\gamma}_{U_{2}}^{n}=\frac{\rho b_{2}\left|g_{n, 2}\right|^{2}}{1+\rho b_{1}\left|g_{n, 2}\right|^{2}}=\frac{b_{2} \gamma_{n, 2}}{1+b_{1} \gamma_{n, 2}},
$$

where $\gamma_{n, i} \triangleq \rho\left|g_{n, i}\right|^{2}, i \in\{1,2\}$ and $\rho=P / N_{0}$ is the transmit SNR at NOMA users.

As for the detection of $s_{2}, U_{1}$ first performs SIC that decodes and removes $U_{2}$ 's message, then proceeds to decode its own message without interference. For this, the instantaneous SINR at $U_{1}$ for the detection of $s_{2}$ is given as:

$$
\tilde{\gamma}_{U_{2} \rightarrow U_{1}}^{n}=\frac{\rho b_{2}\left|g_{n, 1}\right|^{2}}{1+\rho b_{1}\left|g_{n, 1}\right|^{2}}=\frac{b_{2} \gamma_{n, 1}}{1+b_{1} \gamma_{n, 1}},
$$

After the SIC procedure in this context, resulting in a positive residual interference quantity and channel estimation noise without the interference of $s_{1}$ with imperfect SIC (ipSIC) and perfect SIC (pSIC), the instantaneous signal-to-noise ratio (SNR) at $U_{1}$ for the detection of $s_{1}$ is represented as [35]:

$$
\begin{gathered}
\tilde{\gamma}_{U_{1}}^{n, p S I C}=b_{1} \gamma_{n, 1}, \\
\tilde{\gamma}_{U_{1}}^{n, i p S I C}=\frac{b_{1} \gamma_{n, 1}}{1+\rho\left|h_{I}\right|^{2}},
\end{gathered}
$$

where $\left|h_{I}\right|^{2} \sim \mathcal{C N}\left(0, \lambda_{I}\right)$ in which $0 \leq \lambda_{I}<1$ is the level of residual interference caused by imperfect SIC and $\mathcal{C N} \sim(a, b)$ is the complex normal distribution with average $a$ and variance $b$.

\section{ANALYSIS OF OUTAGE PROBABILITY}

\subsection{The channel model}

We set $\gamma_{n}=\gamma_{n_{1}}=\gamma_{n_{2}}$, thus, the probability density function (PDF) of $\gamma_{n}$ is given by [36], [37]:

$$
p_{\gamma_{n}}(x \mid \kappa, \mu, \rho)=\mathcal{G}(\kappa, \mu, \rho) x^{(\mu-1) / 2} e^{-\lambda_{g} x} \mathcal{I}_{\mu-1}\left(2 \sqrt{\kappa \mu \lambda_{g} x}\right) \quad, x>0
$$


where $\lambda_{g}=\frac{(1+\kappa) \mu}{\rho}, \mathcal{G}(\kappa, \mu, \rho)=\frac{\lambda_{g}^{(\mu+1) / 2}}{[\kappa \mu]^{(\mu-1) / 2} e^{\kappa \mu}}, \kappa>0$ designates the total power of dominant components and the total power of scattered waves ratio. $\mu>0$ describes the number of multi-path clusters and $\mathcal{I}_{v}(x)$ denotes the $v$-th order modified Bessel function. Similarly, the PDFs of $\gamma_{i}, i \in\{1,2\}$ in $\kappa-\mu$ fading channels can be expressed by $p_{\gamma_{i}}\left(x_{i} \mid \kappa_{i}, \mu_{i}, \rho\right)$. For arbitrary values $\kappa$ and $\mu$, the cumulative distribution function (CDF) of $\gamma_{n}$ can be obtained as [38], (31).

$$
F_{\gamma_{n}}(x \mid \kappa, \mu, \rho)=1-\mathcal{Q}_{\mu}\left(\sqrt{2 \kappa \mu}, \sqrt{2 \lambda_{g} x}\right) \quad, x>0
$$

with $\mathcal{Q}_{c}(a, b)=a^{1-c} \int_{b}^{\infty} x^{c} e^{-\left(x^{2}+a^{2}\right) / 2} \mathcal{I}_{c-1}(a x) d x$ specifies the generalized Marcum Q-function in [39], (4.33). For integer values of $\mu$ and with the help of [39], (4.63), $F_{\gamma_{n}}(x \mid \kappa, \mu, \rho)$ can be greatly simplified as:

$$
F_{\gamma_{n}}(x \mid \kappa, \mu, \rho)=e^{-\kappa \mu-\lambda_{g} x} \sum_{r=\mu}^{\infty}\left(\frac{\lambda_{g} x}{\kappa \mu}\right)^{r / 2} \mathcal{I}_{r}\left(2 \sqrt{\kappa \mu \lambda_{g} x}\right)
$$

Utilizing $\mathcal{I}_{q}(a \sqrt{x})=\sum_{n=0}^{\infty} \frac{(a \sqrt{x} / 2)^{2 n+r}}{n ! \Gamma(n+r+1)}$ is the modified Bessel function of the first kind of order $q$ in [40], (8.445), we have:

$$
F_{\gamma_{n}}(x \mid \kappa, \mu, \rho)=e^{-\kappa \mu-\lambda_{g} x} \sum_{r=\mu}^{\infty} \sum_{n=0}^{\infty} \frac{\kappa^{n} \mu^{n} \lambda_{g}^{n+r} x^{n+r}}{n ! \Gamma(n+r+1)}
$$

\subsection{Two users outage probability}

When the user's rates are determined based on their channel conditions, OP becomes an important metric for performance evaluation. Hence the performance of two users in terms of the OP is obtained in the following way.

Case 1: $0<\lambda_{I} \leq 1$, we have the OP with ipSIC for $U_{1}$ is calculated as:

$$
\begin{aligned}
\mathcal{P}_{1}^{i p S I C} & =\operatorname{Pr}\left(\max _{n \in N}\left\{\tilde{\gamma}_{U_{2} \rightarrow U_{1}}^{n}\right\}<\tilde{\varepsilon}_{2} \cup \max _{n \in N}\left\{\tilde{\gamma}_{U_{1}}^{n, i p S I C}\right\}<\tilde{\varepsilon}_{1}\right) \\
& =\prod_{n=1}^{N}\left[1-\operatorname{Pr}\left(\tilde{\gamma}_{U_{2} \rightarrow U_{1}}^{n} \geq \tilde{\varepsilon}_{2}, \tilde{\gamma}_{U_{1}}^{n, i p S I C} \geq \tilde{\varepsilon}_{1}\right)\right] \\
& =\left[1-\operatorname{Pr}\left(\gamma_{1} \geq \tilde{\eta}_{2}, \gamma_{1} \geq \tilde{\eta}_{1}\left(1+\rho\left|h_{I}\right|^{2}\right)\right)\right]^{N},
\end{aligned}
$$

where $\tilde{\varepsilon}_{i}=2^{2 R_{i}}-1$, for $i=1,2, R_{i}$ being the target rate at $U_{i}, \tilde{\eta}_{2}=\frac{\tilde{\varepsilon}_{2}}{b_{2}-\tilde{\varepsilon}_{2} b_{1}}$ and $\tilde{\eta}_{1}=\frac{\tilde{\varepsilon}_{1}}{b_{1}}$. Assuming $\tilde{\eta}_{1}\left(1+\rho\left|h_{I}\right|^{2}\right) \gg \tilde{\eta}_{2}, \mathcal{P}_{1}^{i p S I C}$ can be calculated by:

$$
\begin{aligned}
\mathcal{P}_{1}^{i p S I C} & =\left[1-\operatorname{Pr}\left(\gamma_{n, 1} \geq \tilde{\eta}_{1}\left(1+\rho\left|h_{I}\right|^{2}\right)\right)\right]^{N} \\
& =\left\{1-\int_{0}^{\infty} f_{\left|h_{I}\right|^{2}}(x)\left[1-F_{\gamma_{n, 1}}\left(\tilde{\eta}_{1}(1+\rho x)\right)\right] d x\right\}^{N} .
\end{aligned}
$$

\subsubsection{Proposition}

The exact OP with ipSIC at the user $U_{1}$ is written as:

$$
\mathcal{P}_{1}^{i p S I C}=\left[e^{-\kappa \mu-\lambda_{g} \tilde{\eta}_{1}} \sum_{r=\mu}^{\infty} \sum_{n=0}^{\infty} \sum_{q=0}^{n+r}\left(\begin{array}{c}
n+r \\
q
\end{array}\right) \frac{q ! \kappa^{n} \mu^{n} \lambda_{g}^{n+r} \tilde{\eta}_{1}^{n+r} \lambda_{I}^{q} \rho^{q}}{n ! \Gamma(n+r+1)\left(1+\lambda_{I} \lambda_{g} \tilde{\eta}_{1} \rho\right)^{q+1}}\right]^{N},
$$

where $\Gamma(\bullet)$ is the Gamma function [40], (8.310). 


\subsubsection{Proof of proposition}

From $(12), \mathcal{P}_{1}^{i p S I C}$ is expanded is being as:

$$
\begin{aligned}
\mathcal{P}_{1}^{i p S I C} & =\left[1-\int_{0}^{\infty} f_{\left|h_{I}\right|^{2}}(x) d x+\int_{0}^{\infty} f_{\left|h_{I}\right|^{2}}(x) F_{\gamma_{n, 1}}\left(\tilde{\eta}_{1}(1+\rho x)\right) d x\right]^{N} \\
& =\left[1-\lambda_{I} \int_{0}^{\infty} e^{-\frac{x}{\lambda_{I}}} d x+\frac{e^{-\kappa \mu-\lambda_{g} \tilde{\eta}_{1}}}{\lambda_{I}} \sum_{r=\mu}^{\infty} \sum_{n=0}^{\infty} \frac{\kappa^{n} \mu^{n} \lambda_{g}^{n+r} \tilde{\eta}_{1}^{n+r}}{n ! \Gamma(n+r+1)} \int_{0}^{\infty} e^{-x\left(\frac{1}{\lambda_{I}}+\lambda_{g} \tilde{\eta}_{1} \rho\right)}(1+\rho x)^{n+r} d x\right]^{N} .
\end{aligned}
$$

For the first integral, it equals 1. For the second integral we use Newton's Binomial Theorem.

$$
(x+y)^{n}=\sum_{k=0}^{n}\left(\begin{array}{c}
n \\
k
\end{array}\right) x^{n-k} y^{k}=\sum_{k=0}^{n}\left(\begin{array}{c}
n \\
k
\end{array}\right) x^{k} y^{n-k} .
$$

Hence (14) is calculated as:

$$
\mathcal{P}_{1}^{i p S I C}=\frac{e^{-\kappa \mu-\lambda_{g} \tilde{\eta}_{1}}}{\lambda_{I}} \sum_{r=\mu}^{\infty} \sum_{n=0}^{\infty} \sum_{q=0}^{n+r}\left(\begin{array}{c}
n+r \\
q
\end{array}\right) \frac{\kappa^{n} \mu^{n} \lambda_{g}^{n+r} \tilde{\eta}_{1}^{n+r} \rho^{q}}{n ! \Gamma(n+r+1)} \int_{0}^{\infty} e^{-x\left(\frac{1}{\lambda_{I}}+\lambda_{g} \tilde{\eta}_{1} \rho\right)} x^{q} d x .
$$

Using [40], (3.351.3), we have close-form outage probability with ipSIC of $U_{1}$ given as:

$$
\mathcal{P}_{1}^{i p S I C}=\left[e^{-\kappa \mu-\lambda_{g} \tilde{\eta}_{1}} \sum_{r=\mu}^{\infty} \sum_{n=0}^{\infty} \sum_{q=0}^{n+r}\left(\begin{array}{c}
n+r \\
q
\end{array}\right) \frac{q ! \kappa^{n} \mu^{n} \lambda_{g}^{n+r} \tilde{\eta}_{1}^{n+r} \lambda_{I}^{q} \rho^{q}}{n ! \Gamma(n+r+1)\left(1+\lambda_{I} \lambda_{g} \tilde{\eta}_{1} \rho\right)^{q+1}}\right]^{N} .
$$

Based on the aforementioned results, the proof is complete.

Case 2: $\lambda_{I}=0, \mathcal{P}_{1}^{p S I C}$ is given as:

$$
\begin{aligned}
\mathcal{P}_{1}^{p S I C} & =\operatorname{Pr}\left(\max _{n \in N}\left\{\tilde{\gamma}_{U_{2} \rightarrow U_{1}}^{n}\right\}<\tilde{\varepsilon}_{2} \cup \max _{n \in N}\left\{\tilde{\gamma}_{U_{1}}^{n, p S I C}\right\}<\tilde{\varepsilon}_{1}\right) \\
& =\prod_{n=1}^{N}\left[1-\operatorname{Pr}\left(\gamma_{n, 1} \geq \tilde{\eta}_{2}, \gamma_{n, 1} \geq \tilde{\eta}_{1}\right)\right] \\
& =\left[1-\operatorname{Pr}\left(\gamma_{n, 1} \geq \tilde{\eta}_{\max }\right)\right]^{N} \\
& =\left[F_{\gamma_{n, 1}}\left(\tilde{\eta}_{\max }\right)\right]^{N},
\end{aligned}
$$

where $\tilde{\eta}_{\max }=\left[\tilde{\eta}_{1}, \tilde{\eta}_{2}\right]^{+}$in which $[a, b]^{+}=\max (a, b)$.

Applying (8), (10), and (18) can be calculated as:

$$
\begin{aligned}
\mathcal{P}_{1}^{p S I C} \stackrel{\mu \in \mathbb{Q}}{=} & {\left[\mathcal{Q}_{\mu}\left(\sqrt{2 \kappa \mu}, \sqrt{2 \lambda_{g} \tilde{\eta}_{\max }}\right)\right]^{N} } \\
\stackrel{\mu \in \mathbb{N}}{=} & {\left[e^{-\kappa \mu-\lambda_{g} \tilde{\eta}_{\max }} \sum_{r=\mu}^{\infty} \sum_{n=0}^{\infty} \frac{\kappa^{n} \mu^{n} \lambda_{g}^{n+r} \tilde{\eta}_{\max }^{n+r}}{n ! \Gamma(n+r+1)}\right]^{N} . }
\end{aligned}
$$

Solving of $\mathcal{P}_{1}^{p S I C}$ we obtain the closed-form OP at $U_{2}$ as:

$$
\begin{aligned}
\mathcal{P}_{2} & =\operatorname{Pr}\left(\max _{n \in N}\left\{\tilde{\gamma}_{U_{2}}^{n}\right\}<\tilde{\varepsilon}_{2}\right) \\
& =\prod_{n=1}^{N}\left[1-\operatorname{Pr}\left(\tilde{\gamma}_{U_{2}}^{n} \geq \tilde{\varepsilon}_{2}\right)\right] \\
& =\left[1-\operatorname{Pr}\left(\gamma_{n, 2} \geq \tilde{\eta}_{2}\right)\right]^{N} \\
& =\left[F_{\gamma_{n, 2}}\left(\tilde{\eta}_{2}\right)\right]^{N}
\end{aligned}
$$


Similarly, we obtain the OP of $U_{2}$ as:

$$
\begin{aligned}
\mathcal{P}_{2} & \stackrel{\mu \in \mathbb{Q}}{=}\left[\mathcal{Q}_{\mu}\left(\sqrt{2 \kappa \mu}, \sqrt{2 \lambda_{g} \tilde{\eta}_{2}}\right)\right]^{N} \\
& \stackrel{\mu \in \mathbb{N}}{=}\left[e^{-\kappa \mu-\lambda_{g} \tilde{\eta}_{2}} \sum_{r=\mu}^{\infty} \sum_{n=0}^{\infty} \frac{\kappa^{n} \mu^{n} \lambda_{g}^{n+r} \tilde{\eta}_{2}^{n+r}}{n ! \Gamma(n+r+1)}\right]^{N} .
\end{aligned}
$$

\section{NUMERICAL RESULTS}

In this section, $\kappa-\mu$ fading is used; see Table I for a list of examples. We use mathematical derivations to mimic OP and then use Monte-Carlo simulation to check these derivations. Table 2 contains a list of the parameters.

Table 1. The $\kappa-\mu$ distribution yields common fading distributions [41]

\begin{tabular}{cc}
\hline Channels & $\kappa-\mu$ Distribution parameters \\
\hline Rayleigh & $\mu=1, \kappa \rightarrow 0$ \\
One-sided Gaussian & $\mu=0.5, \kappa \rightarrow 0$ \\
$\kappa-\mu$ & $\mu=\mu, \kappa=\kappa$ \\
Nakagami- $m$ & $\mu=m, \kappa \rightarrow 0$ \\
Rician with parameter $K$ & $\mu=1, \kappa=K$ \\
\hline
\end{tabular}

Table 2. In the performance evaluation, system parameters were employed [42]

\begin{tabular}{cc}
\hline System Parameters & Values \\
\hline Monte Carlo simulations repeated & $10^{6}$ iterations \\
Antennas of BS & $N=2$ \\
The power allocation coefficients & $\left\{b_{1}, b_{2}\right\}=\{0.1,0.9\}$ \\
The target rate at $U_{1}$ & $R_{1}=2 \mathrm{bps} / \mathrm{Hz}$ \\
The target rate at $U_{2}$ & $R_{2}=1 \mathrm{bps} / \mathrm{Hz}$ \\
The interference signal's effect level (IS) & $\lambda_{I}=0.001$ \\
\hline
\end{tabular}

Figure 2 depicts OP for user $U_{1}$ with imperfect SIC versus transmit SNR for different fading channels. From the Figure, we can observe how the level of interference signal $\lambda_{I}$ impacts OP. The best OP performance is achieved under Nakagami- $m$ fading channels for both cases of $\lambda_{I}$. Also, the OP curves approach a floor at large SNR values as this is indicative of the error introduced by SIC in general. However, the OP performance curves have different values depending on the $\lambda_{I}$ value. The reason is that imperfect SIC under performs in cases of large levels of interference signal as to be expected from the literature.

Figure 3 depicts OP for both user $U_{1}$ with perfect and imperfect SIC conditions, and user $U_{2}$ versus transmit SNR for different fading channels with $\lambda_{I}=0.001$. From the Figure, we can observe how the different values of $\kappa-\mu$ distribution impact OP. The best OP performance is achieved under $(\kappa-\mu)=(2,0)$ for both $U_{1}$ and $U_{2}$. In addition, we can observe that OP for user $U_{2}$ does not approach a floor as SIC is not utilized at the user receiver but the OP curves for $U_{1}$ approach a floor at large SNR values when perfect SIC is exploited at the receiver.

Figure 4 depicts OP for both user $U_{1}$ with perfect and imperfect SIC conditions, and user $U_{2}$ versus power allocation $b_{2}$ for different values of fading channels with target rates $R_{1}=R_{2}=0.5, \lambda_{I}=0.01$ and $\rho=15 \mathrm{~dB}$. From the Figure, we can observe how the different values of $\kappa-\mu$ distribution impact OP. The best OP performance is achieved under $(\kappa-\mu)=(2,0)$ for both $U_{1}$ and $U_{2}$. Moreover, at about $b_{2}=0.65$ the OP for both SIC cases for user $U_{1}$ starts performing poorly. This demonstrates the relationship between power allocation coefficients and $\mathrm{OP}$.

Figure 5 depicts OP for both user $U_{1}$ with perfect and imperfect SIC conditions, and user $U_{2}$ versus varying target rates $R_{1}=R_{2}, \lambda_{I}=0.01, b_{1}=0.05, b_{2}=0.95$ and while varying $\rho \mathrm{dB}$. In Figure 5 , we can see how the different values of $R_{1}=R_{2}$ and $\rho$ impact OP. The best OP performance is achieved at $R_{1}=R_{2}=0.3$ bit/s/Hz for both $U_{1}$ and $U_{2}$. Also, for user $U_{1}$ the OP hits a ceiling at high $R_{1}=R_{2}$ values. 


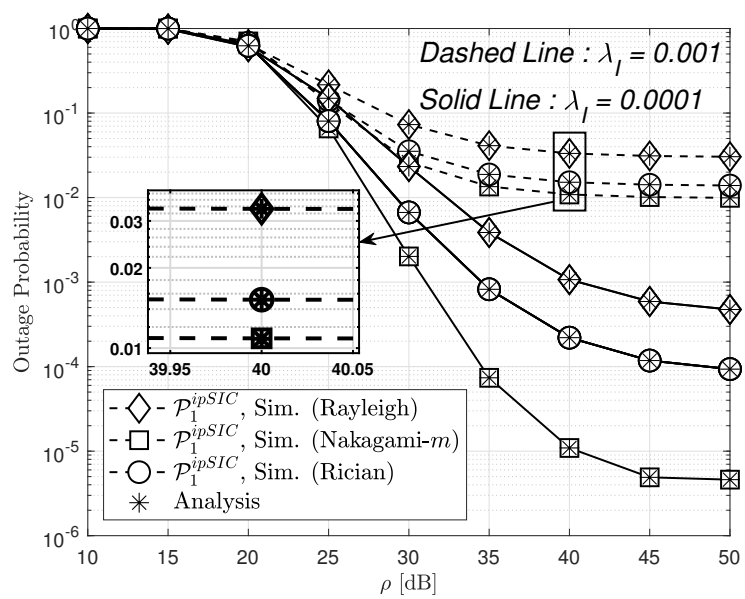

Figure 2. Probability of $U_{1}$ with ipSIC versus $\rho$ [dB] for different fading channels

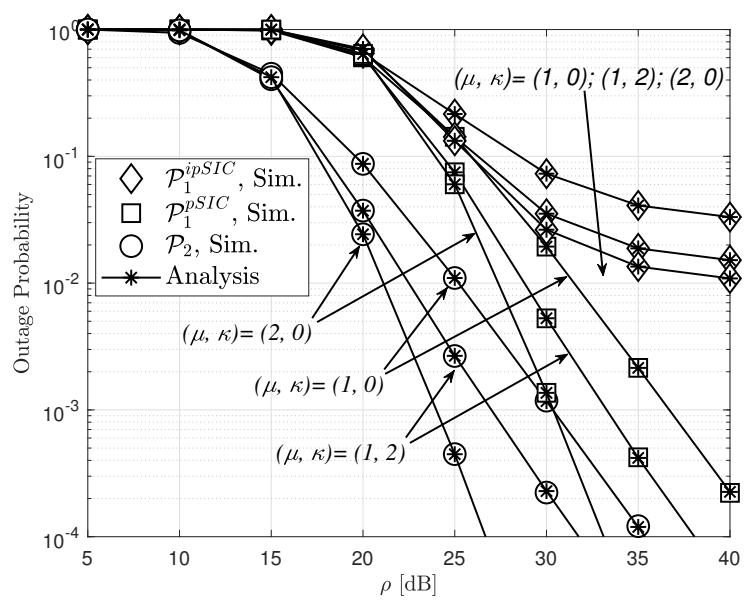

Figure 3. Outage probability versus $\rho[\mathrm{dB}]$ for different fading channels, with $\lambda_{I}=0.001$

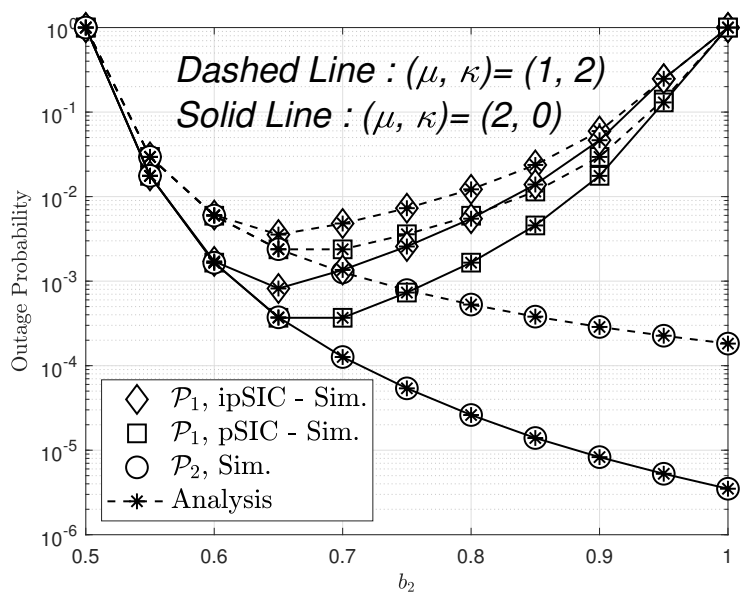

Figure 4. Outage probability versus $b_{2}$ for different values of general fading channel, with $R_{1}=R_{2}=0.5$, $\lambda_{I}=0.01$ and $\rho=15[\mathrm{~dB}]$ 


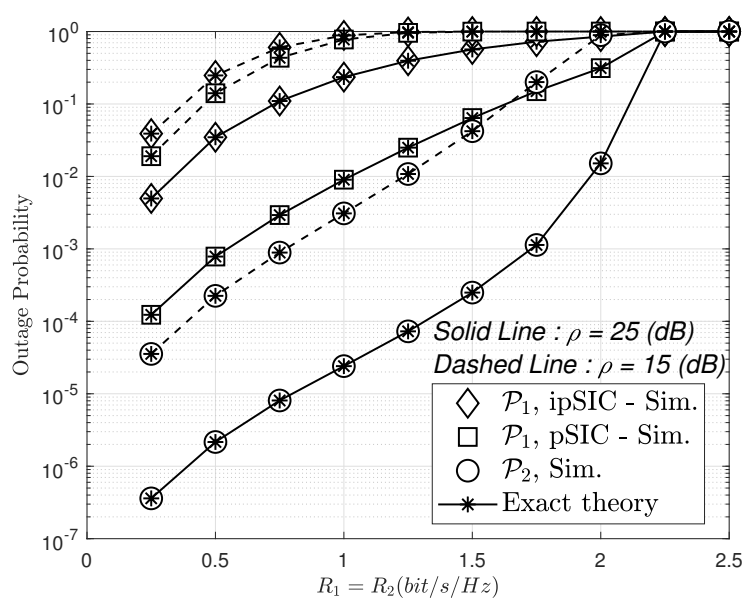

Figure 5. Outage probability versus target rate, with $\lambda_{I}=0.01, b_{1}=0.05$ and $b_{2}=0.95$

\section{CONCLUSION}

We studied a NOMA communication network with two users $D_{1}$ and $D_{2}$ in this study. We also developed mathematical formulae for OP in the case of a receiver with an incomplete SIC. These expressions were simulated using different $\kappa-\mu$ fading distribution channels (which are more generic than particular situations like Rayleigh, Nakagami- $m$, and Rician fading channels). The additional simulations were done using the general fading distribution of $\kappa$ and $\mu$ as the major discovery. As can be shown from the simulations, imperfect SIC outperforms flawless SIC models.

\section{ACKNOWLEDGEMENTS}

The research leading to this results was supported by Czech Ministry of Education, Youth and Sports under project reg. no. SP2021/25 and also partially under the e-INFRA CZ project ID:90140.

\section{REFERENCES}

[1] L. Dai, B. Wang, Y. Yuan, S. Han, I. Chih-lin, and Z. Wang, "Non-orthogonal multiple access for 5G: solutions, challenges, opportunities, and future research trends," in IEEE Communications Magazine, vol. 53, no. 9, pp. 74-81, September 2015, doi: 10.1109/MCOM.2015.7263349.

[2] Y. Liu, Z. Qin, M. Elkashlan, Z. Ding, A. Nallanathan, and L. Hanzo, "Nonorthogonal Multiple Access for 5G and Beyond," in Proceedings of the IEEE, vol. 105, no. 12, pp. 2347-2381, Dec. 2017, doi: 10.1109/JPROC.2017.2768666.

[3] H. Sadia, M. Zeeshan, and S. A. Sheikh, "Performance analysis of downlink power domain NOMA under fading channels," 2018 ELEKTRO, 2018, pp. 1-6, doi: 10.1109/ELEKTRO.2018.8398247.

[4] Y. Saito, Y. Kishiyama, A. Benjebbour, T. Nakamura, A. Li, and K. Higuchi, "Non-Orthogonal Multiple Access (NOMA) for Cellular Future Radio Access," 2013 IEEE 77th Vehicular Technology Conference (VTC Spring), Dresden, Germany, 2013, pp. 1-5, doi: 10.1109/VTCSpring.2013.6692652.

[5] S. M. R. Islam, N. Avazov, O. A. Dobre, and K. Kwak, "Power-Domain Non-Orthogonal Multiple Access (NOMA) in 5G Systems: Potentials and Challenges," in IEEE Communications Surveys \& Tutorials, vol. 19, no. 2, pp. 721-742, Secondquarter 2017, doi: 10.1109/COMST.2016.2621116.

[6] Z. Ding, X. Lei, G. K. Karagiannidis, R. Schober, J. Yuan, and V. K. Bhargava, ”A Survey on Non-Orthogonal Multiple Access for 5G Networks: Research Challenges and Future Trends," in IEEE Journal on Selected Areas in Communications, vol. 35, no. 10, pp. 2181-2195, Oct. 2017, doi: 10.1109/JSAC.2017.2725519.

[7] Z. Ding et al., "Application of Non-Orthogonal Multiple Access in LTE and 5G Networks," in IEEE Communications Magazine, vol. 55, no. 2, pp. 185-191, February 2017, doi: 10.1109/MCOM.2017.1500657CM.

[8] M. S. Ali, H. Tabassum, and E. Hossain, ”Dynamic User Clustering and Power Allocation for Uplink and Downlink Non-Orthogonal Multiple Access (NOMA) Systems," in IEEE Access, vol. 4, pp. 6325-6343, 2016, doi: 10.1109/ACCESS.2016.2604821.

[9] K. Senel, H. V. Cheng, E. Björnson, and E. G. Larsson, "What Role can NOMA Play in Massive MIMO?," in IEEE Journal of Selected Topics in Signal Processing, vol. 13, no. 3, pp. 597-611, June 2019, doi: 10.1109/JSTSP.2019.2899252.

[10] Y. Sun, Z. Ding, and X. Dai, "On the Performance of Downlink NOMA in Multi-Cell mmWave Networks," in IEEE Communications Letters, vol. 22, no. 11, pp. 2366-2369, Nov. 2018, doi: 10.1109/LCOMM.2018.2870442.

[11] X. Zhang, et al., "Outage Performance of NOMA-Based Cognitive Hybrid Satellite-Terrestrial Overlay Networks by Amplify-andForward Protocols," in IEEE Access, vol. 7, pp. 85372-85381, 2019, doi: 10.1109/ACCESS.2019.2925314.

[12] Y. Zhang, K. Peng, Z. Chen, and J. Song, "SIC vs. JD: Uplink NOMA techniques for M2M random access," 2017 IEEE International Conference on Communications (ICC), 2017, pp. 1-6, doi: 10.1109/ICC.2017.7996497. 
[13] Y. Liu, Z. Qin, M. Elkashlan, Y. Gao, and A. Nallanathan, "Non-Orthogonal Multiple Access in Massive MIMO Aided Heterogeneous Networks," 2016 IEEE Global Communications Conference (GLOBECOM), 2016, pp. 1-6, doi: 10.1109/GLOCOM.2016.7842027

[14] E. G. Larsson, O. Edfors, F. Tufvesson, and T. L. Marzetta, "Massive MIMO for next generation wireless systems," in IEEE Communications Magazine, vol. 52, no. 2, pp. 186-195, February 2014, doi: 10.1109/MCOM.2014.6736761.

[15] G. Liu, et al., "3-D-MIMO With Massive Antennas Paves the Way to 5G Enhanced Mobile Broadband: From System Design to Field Trials," in IEEE Journal on Selected Areas in Communications, vol. 35, no. 6, pp. 1222-1233, June 2017, doi: 10.1109/JSAC.2017.2687998.

[16] M. Zeng, A. Yadav, O. A. Dobre, G. I. Tsiropoulos, and H. V. Poor, "Capacity Comparison Between MIMO-NOMA and MIMOOMA With Multiple Users in a Cluster," in IEEE Journal on Selected Areas in Communications, vol. 35, no. 10, pp. 2413-2424, Oct. 2017, doi: 10.1109/JSAC.2017.2725879.

[17] Y. Liu, M. Elkashlan, Z. Ding, and G. K. Karagiannidis, "Fairness of User Clustering in MIMO Non-Orthogonal Multiple Access Systems," in IEEE Communications Letters, vol. 20, no. 7, pp. 1465-1468, July 2016, doi: 10.1109/LCOMM.2016.2559459.

[18] J. Men and J. Ge, "Non-Orthogonal Multiple Access for Multiple-Antenna Relaying Networks," in IEEE Communications Letters, vol. 19, no. 10, pp. 1686-1689, Oct. 2015, doi: 10.1109/LCOMM.2015.2472006.

[19] Q. Sun, S. Han, C. I, and Z. Pan, "On the Ergodic Capacity of MIMO NOMA Systems," in IEEE Wireless Communications Letters, vol. 4, no. 4, pp. 405-408, Aug. 2015, doi: 10.1109/LWC.2015.2426709.

[20] M. F. Hanif, Z. Ding, T. Ratnarajah, and G. K. Karagiannidis, "A Minorization-Maximization Method for Optimizing Sum Rate in the Downlink of Non-Orthogonal Multiple Access Systems," in IEEE Transactions on Signal Processing, vol. 64, no. 1, pp. 76-88, Jan.1, 2016, doi: 10.1109/TSP.2015.2480042.

[21] W. Shin, M. Vaezi, B. Lee, D. J. Love, J. Lee, and H. V. Poor, "Coordinated Beamforming for Multi-Cell MIMO-NOMA," in IEEE Communications Letters, vol. 21, no. 1, pp. 84-87, Jan. 2017, doi: 10.1109/LCOMM.2016.2615097.

[22] Z. Ding, R. Schober, and H. V. Poor, "A General MIMO Framework for NOMA Downlink and Uplink Transmission Based on Signal Alignment," in IEEE Transactions on Wireless Communications, vol. 15, no. 6, pp. 4438-4454, June 2016, doi: 10.1109/TWC.2016.2542066.

[23] M. Zeng, A. Yadav, O. A. Dobre, G. I. Tsiropoulos, and H. V. Poor, "On the Sum Rate of MIMO-NOMA and MIMO-OMA Systems," in IEEE Wireless Communications Letters, vol. 6, no. 4, pp. 534-537, Aug. 2017, doi: 10.1109/LWC.2017.2712149.

[24] S. Ali, E. Hossain, and D. I. Kim, "Non-Orthogonal Multiple Access (NOMA) for Downlink Multiuser MIMO Systems: User Clustering, Beamforming, and Power Allocation," in IEEE Access, vol. 5, pp. 565-577, 2017, doi: 10.1109/ACCESS.2016.2646183.

[25] D. Zhang, Z. Zhou, C. Xu, Y. Zhang, J. Rodriguez, and T. Sato, "Capacity Analysis of NOMA With mmWave Massive MIMO Systems," in IEEE Journal on Selected Areas in Communications, vol. 35, no. 7, pp. 1606-1618, July 2017, doi: 10.1109/JSAC.2017.2699059.

[26] C.-B. Le, D.-T. Do, X. Li, Y.-F. Huang, H.-C. Chen, and M. Voznak, "Enabling NOMA in backscatter reconfigurable intelligent surfaces-aided systems," in IEEE Access, vol. 9, pp. 33782-33795, Feb. 2021, doi: 10.1109/ACCESS.2021.3061429.

[27] Y. Li and G. A. Aruma Baduge, "NOMA-Aided Cell-Free Massive MIMO Systems," in IEEE Wireless Communications Letters, vol. 7, no. 6, pp. 950-953, Dec. 2018, doi: 10.1109/LWC.2018.2841375.

[28] A. A. Ohashi, et al., "Cell-Free Massive MIMO-NOMA Systems With Imperfect SIC and Non-Reciprocal Channels," in IEEE Wireless Communications Letters, vol. 10, no. 6, pp. 1329-1333, June 2021, doi: 10.1109/LWC.2021.3066042.

[29] Y. Zhang, H. Cao, M. Zhou, and L. Yang, "Non-orthogonal multiple access in cell-free massive MIMO networks," in China Communications, vol. 17, no. 8, pp. 81-94, Aug. 2020, doi: 10.23919/JCC.2020.08.007.

[30] X. Li, J. Li, Y. Liu, Z. Ding, and A. Nallanathan, "Residual Transceiver Hardware Impairments on Cooperative NOMA Networks," in IEEE Transactions on Wireless Communications, vol. 19, no. 1, pp. 680-695, Jan. 2020, doi: 10.1109/TWC.2019.2947670

[31] N. P. Le, et al., "Performance Analysis of Uplink NOMA Systems with Imperfect SIC Detection and Delay Constraints over Composite Fading Channels," in IEEE Transactions on Vehicular Technology, doi: 10.1109/TVT.2021.3086045.

[32] A. Agarwal, R. Chaurasiya, S. Rai, and A. K. Jagannatham, "Outage Probability Analysis for NOMA Downlink and Uplink Communication Systems With Generalized Fading Channels," in IEEE Access, vol. 8, pp. 220461-220481, 2020, doi: 10.1109/ACCESS.2020.3042993.

[33] B. M. ElHalawany, F. Jameel, D. B. da Costa, U. S. Dias, and K. Wu, "Performance Analysis of Downlink NOMA Systems Over $\kappa-\mu$ Shadowed Fading Channels," in IEEE Transactions on Vehicular Technology, vol. 69, no. 1, pp. 1046-1050, Jan. 2020, doi: 10.1109/TVT.2019.2953109.

[34] Chi-Bao Le and Dinh-Thuan Do, "Joint evaluation of imperfect SIC and fixed power allocation scheme for wireless powered D2D-NOMA networks with multiple antennas at base station," Wireless Network, vol. 25, no. 8, pp. 5069-5081, Aug. 2019, doi: 10.1007/s11276-019-02116-1.

[35] D. Do, T. Anh Le, T. N. Nguyen, X. Li, and K. M. Rabie, "Joint impacts of imperfect CSI and imperfect SIC in cognitive radio assisted NOMA-V2X communications," IEEE Access, vol. 8, pp. 128629-128645, 2020, doi: 10.1109/ACCESS.2020.3008788.

[36] M. D. Yacoub, "The $\kappa-\mu$ distribution and the $\eta-\mu$ distribution," IEEE Anten. Prop. Mag., vol. 49, no. 1, pp. 68-81, Feb. 2007, doi: 10.1109/MAP.2007.370983.

[37] W. Li and M. Chen, "Outage capacity of dual-hop decode-and-forward relaying system over generalized fading channels," 20102 nd International Conference on Future Computer and Communication, 2010, pp. V3-827-V3-831, doi: 10.1109/ICFCC.2010.5497387.

[38] N. Y. Ermolova and O. Tirkkonen, "Outage Probability Analysis in Generalized Fading Channels with Co-Channel Interference and Background Noise: $\eta-\mu / \eta-\mu, \eta-\mu / \kappa-\mu$ and $\kappa-\mu / \eta-\mu$ Scenarios," IEEE Trans. Wireless Commun., vol. 13, no. 1, pp. 291-297, January 2014, doi: 10.1109/TWC.2013.110813.130624.

[39] M. K. Simon and M.-S. Alouini, Digital Communication over Fading Channels, John Wiley \& Sons, vol. $95,2005$.

[40] I. S. Gradshteyn and I. M. Ryzhik, Tables of Integrals, Series and Products, 6th ed. New York: Academic Press, 2000.

[41] J. F. Paris, "Statistical Characterization of $\kappa-\mu$ Shadowed Fading," IEEE Trans. Veh. Technol., vol. 63, no. 2, pp. 518-526, Feb 2014, doi: 10.1109/TVT.2013.2281213.

[42] D.-T. Do, A.-T. Le, and B. M. Lee, "NOMA in cooperative underlay cognitive radio networks under imperfect SIC," IEEE Access, vol. 8, pp. 86180-86195, 2020, doi: 10.1109/ACCESS.2020.2992660. 


\section{BIOGRAPHIES OF AUTHORS}

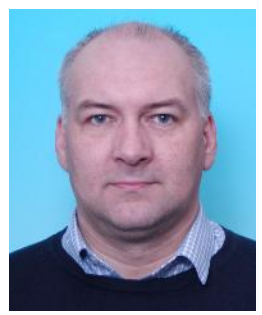

Miroslav Voznak (10) If 5 (M'09-SM' 16) received his PhD in telecommunications in 2002 from the Faculty of Electrical Engineering and Computer Science at VSB - Technical University of Ostrava, and achieved habilitation in 2009. He was appointed Full Professor in Electronics and Communications Technologies in 2017. His research interests generally focus on ICT, especially on quality of service and experience, network security, wireless networks, and big data analytics. He has authored and co-authored over one hundred articles in SCI/SCIE journals. According to the Stanford University study released in 2020, he is one of the World's Top 2 percent of scientists in Networking Telecommunications and Information Communications Technologies. He served as a general chair of the 11th IFIP Wireless and Mobile Networking Conference in 2018 and the 24th IEEE/ACM International Symposium on Distributed Simulation and Real Time Applications in 2020. He participated in six projects funded by the EU in programs managed directly by European Commission. Currently, he is a principal investigator in the research project QUANTUM5 funded by NATO, which focuses on the application of quantum cryptography in $5 \mathrm{G}$ campus networks. He can be contacted at email: mirolav.voznak@vsb.cz.

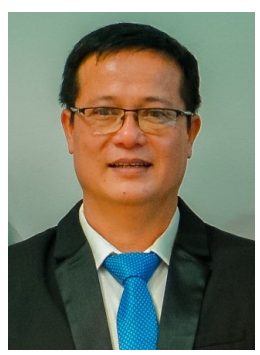

Hong-Nhu Nguyen (D) 8 . 5 P was born in Tien Giang Province, Vietnam, in 1971. He received a B.Sc. in Electronics Engineering from Ho Chi Minh city University of Technology in 1998 and an M.Sc. in Electronics Engineering from the University of Transport and Communications (Vietnam) in 2012. He is currently working as lecturer at Saigon University. He received his $\mathrm{PhD}$ in telecommunication from Technical University of Ostrava, Czech Republic in 2021. His research interest include applied electronics, wireless communications, cognitive radio, NOMA and energy harvesting. He can be contacted at email: nhu.nh@sgu.edu.vn.

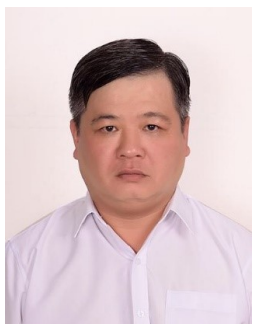

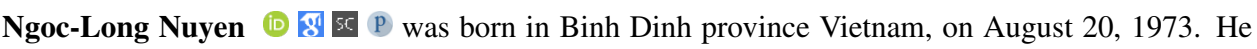
received B.S. and M.S. degrees in Electric Physics of the University of Science (Vietnam). He is currently working as a lecturer at the Faculty of Applied Sciences of Ton Duc Thang University. $\mathrm{He}$ received $\mathrm{PhD}$ degree at Technical University of Ostrava, Czech Republic. His research interest includes applied electronics, satellite system, NOMA. He can be contacted at email: nguyenngoclong@tdtu.edu.vn.

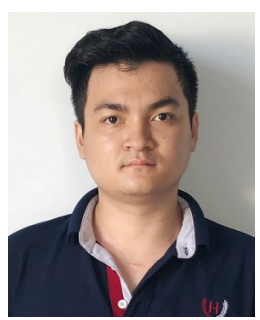

Chi-Bao Le (D) 8 sc $\mathrm{P}$ was born in Binh Thuan, Vietnam. He is currently pursuing the master's degree in wireless communications. He has worked closely with Dr. Thuan at the Wireless Communications and Signal Processing Research Group, Industrial University of Ho Chi Minh City, Vietnam. His research interests include electronic design, signal processing in wireless communications networks, non-orthogonal multiple access, and physical layer security. He can be contacted at email: lechibao@iuh.edu.vn.

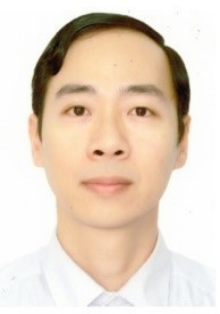

Nhan Duc Nguyen (10) 5 S P received M.Eng in electronic materials from International Training Institute for Materials Science (ITIMS), Hanoi University of Technology in 1998 and his PhD degree in electrical and computer systems engineering from Monash University, Australia in 2011. He had joined the Faculty of Telecommunications, Post and Telecommunication Institute of Technology in Vietnam as a lecturer since 1999. He served as the Head of Signals and Systems Department at Post and Telecommunication Institute of Technology from 2014 to 2020 . He is currently serving as a Systems Engineering Director of the Innovation Center, Van Lang University. His research interests focus on optical communications, numerical modeling and analysis, signal processing, and sensor data processing in machine learning. He can be contacted at email: nhan.nd@ vlu.edu.vn. 